\title{
ORTHOGONAL POLYNOMIALS AND CUBATURE FORMULAE ON SPHERES AND ON SIMPLICES
}

\author{
YUAN XU
}

\begin{abstract}
Orthogonal polynomials on the standard simplex $\Sigma^{d}$ in $\mathbb{R}^{d}$ are shown to be related to the spherical orthogonal polynomials on the unit sphere $S^{d}$ in $\mathbb{R}^{d+1}$ that are invariant under the group $\mathbb{Z}_{2} \times \cdots \times \mathbb{Z}_{2}$. For a large class of measures on $S^{d}$ cubature formulae invariant under $\mathbb{Z}_{2} \times \cdots \times \mathbb{Z}_{2}$ are shown to be characterized by cubature formulae on $\Sigma^{d}$. Moreover, it is also shown that there is a correspondence between orthogonal polynomials and cubature formulae on $\Sigma^{d}$ and those invariant on the unit ball $B^{d}$ in $\mathbb{R}^{d}$. The results provide a new approach to study orthogonal polynomials and cubature formulae on spheres and on simplices.
\end{abstract}

\section{INTRODUCTION}

The purpose of this paper is to study the connection between orthogonal polynomials and cubature formulae on spheres and on simplices. A special case of the results shows that the orthogonal polynomials with respect to the measure $\left(u_{1} \ldots u_{d}\left(1-u_{1}-\ldots-\right.\right.$ $\left.\left.u_{d}\right)\right)^{-1 / 2} d \mathbf{u}$ on the standard simplex $\Sigma^{d}$ on $\mathbb{R}^{d}$ are related to the spherical harmonics on the unit sphere $S^{d}$ in $\mathbb{R}^{d+1}$ that are invariant under the group $\mathbb{Z}_{2} \times \cdots \times \mathbb{Z}_{2}$, and there is a one-to-one correspondence between cubature formulae on $\Sigma^{d}$ and cubature formulae on $S^{d}$ that are invariant under $\mathbb{Z}_{2} \times \cdots \times \mathbb{Z}_{2}$. The main results will be established for a large class of measures. Orthogonal polynomials on the sphere with respect to a measure other than the surface measure have been studied only recently (see $[3,4,5,8,25]$ and the references there). The most important development has been a theory developed by Dunkl for measures invariant under a finite reflection group, in which the role of Laplacian operator is replaced by a differential-difference operator in the commutative algebra generated by a family of commuting first order differential-difference operators (Dunkl's operators). Motivated by the work of Dunkl, in [25] we used an elementary approach to study the connection between orthogonal polynomials and cubature formulae on the sphere $S^{d}$ and the unit ball $B^{d}$ of $\mathbb{R}^{d}$. It was shown that we can construct homogeneous orthogonal polynomials on $S^{d}$ from the corresponding orthogonal polynomials on $B^{d}$ for a large class of measures.

1991 Mathematics Subject Classification. 33C50, 33C55, 65D32.

Key words and phrases. Orthogonal polynomials in several variables, on spheres, on simplices, on balls, cubature formulae.

Supported by the National Science Foundation under Grant DMS-9500532.

Typeset by $\mathcal{A M}_{\mathcal{M}}-\mathrm{T}_{\mathrm{E}} \mathrm{X}$ 
The present work is a continuation of [25] in spirit. For a large class of measures we will show that orthogonal polynomials on $\Sigma^{d}$ and those on $S^{d}$ which are invariant under the group $G=\mathbb{Z}_{2} \times \cdots \times \mathbb{Z}_{2}$ are connected by a simple transformation. The result offers a way to study the structure of orthogonal polynomials on the sphere; it may also lead to new understanding on orthogonal polynomials on the simplex. Together with the results in [25], we also obtain a correspondence between orthogonal polynomials on the simplex and those on the unit ball that are invariant under $G$. Our approach is elementary as in [25]; it is motivated by [3] in which the connection between orthogonal polynomials on $S^{2}$ and $\Sigma^{2}$ is used to study orthogonal polynomials with symmetry of order three; the approach does not involve differential or differential-difference operators.

A closely related question is constructing cubature formulae on spheres and on simplices. According to a theorem of Sobolev, in order to establish a cubature formula invariant under a finite group, one only has to verify those polynomials that are invariant under the same group. In particular, to establish a $\mathbb{Z}_{2} \times \cdots \times \mathbb{Z}_{2}$ invariant cubature formula on $S^{d}$, we only have to consider the homogeneous polynomials that are invariant under the group $G$. This consideration leads us to establish a one-to-one correspondence between the cubature formulae on $\Sigma^{d}$ and the $\mathbb{Z}_{2} \times \cdots \times \mathbb{Z}_{2}$ invariant cubature formula on $S^{d}$. Moreover, using the result in [25], we also have a correspondence between the cubature formulae on $\Sigma^{d}$ and the $\mathbb{Z}_{2} \times \cdots \times \mathbb{Z}_{2}$ invariant cubature formulae on $B^{d}$. These results allow us to transform cubature formulae on one domain to another. Over the years, there has been a lot of efforts devoted to the construction of cubature formulae on the sphere, on the ball and on the simplex (mostly with respect to the Lebesgue measure); see $[6,12,15,17]$ for some of the references. It is remarkable that the simple correspondence between cubature formulae on these regions has not been noticed before. It yields, in particular, many new cubature formulae on spheres and on simplices; some of them can be derived from the known cubature formulae on a different domain by the correspondence. Because the main focus of this paper is on the relation between the orthogonal structure and cubature formulae on spheres and on simplices, we will concentrate on the theoretic side of the matter. The examples of new cubature formulae will be reported in a separate paper $([7])$.

The paper is organized as follows. In Section 2 we introduce notation and present the necessary preliminaries, where we also prove the basic lemma. In Section 3 we discuss the relation between orthogonal polynomials on $S^{d}$ and those on $\Sigma^{d}$. In Section 4 we discuss the relation between cubature formulae on the unit sphere and those on the simplex.

\section{Preliminary and Basic Lemma}

Basic notation. For $\mathbf{x} \in \mathbb{R}^{d}$ we denote by $|\mathbf{x}|=\sqrt{x_{1}^{2}+\ldots+x_{d}^{2}}$ the usual Euclidean norm. Let $B^{d}$ be the unit ball of $\mathbb{R}^{d}$ and $S^{d}$ be the unit sphere on $\mathbb{R}^{d+1}$; that is,

$$
B^{d}=\left\{\mathbf{x} \in \mathbb{R}^{d}:|\mathbf{x}| \leq 1\right\} \quad \text { and } \quad S^{d}=\left\{\mathbf{y} \in \mathbb{R}^{d+1}:|\mathbf{y}|=1\right\} .
$$

We also denote the $\ell^{1}$ norm of $\mathbf{x} \in \mathbb{R}^{d}$ by $|\mathbf{x}|_{1}=\left|x_{1}\right|+\ldots+\left|x_{d}\right|$. Let $\Sigma^{d}$ be the standard simplex in $\mathbb{R}^{d}$; that is,

$$
\Sigma^{d}=\left\{\mathbf{u} \in \mathbb{R}^{d}: u_{1} \geq 0, \ldots, u_{d} \geq 0,1-|\mathbf{u}|_{1} \geq 0\right\} .
$$


For $d=2$, the simplex $\Sigma^{2}$ is the triangle with vertices at $(0,0),(1,0)$ and $(0,1)$. Throughout this paper we fix the following notation: For $\mathbf{y} \in \mathbb{R}^{d+1}$, we write

$$
\mathbf{y}=\left(y_{1}, \ldots, y_{d}, y_{d+1}\right)=r \mathbf{x}^{\prime}=r\left(\mathbf{x}, x_{d+1}\right), \quad \mathbf{x}^{\prime} \in S^{d}, \quad \mathbf{x} \in B^{d},
$$

where $r=|\mathbf{y}|=\sqrt{y_{1}^{2}+\cdots+y_{d+1}^{2}}$ and $\mathbf{x}=\left(x_{1}, \ldots, x_{d}\right)$.

Polynomial spaces. Let $\mathbb{N}_{0}$ be the set of nonnegative integers. For $\alpha=\left(\alpha_{1}, \ldots, \alpha_{d}\right) \in$ $\mathbb{N}_{0}^{d}$ and $\mathbf{x}=\left(x_{1}, \ldots, x_{d}\right) \in \mathbb{R}^{d}$ we write $\mathbf{x}^{\alpha}=x_{1}^{\alpha_{1}} \cdots x_{d}^{\alpha_{d}}$. The number $|\alpha|_{1}=\alpha_{1}+\cdots+\alpha_{d}$ is called the total degree of $\mathbf{x}^{\alpha}$. We denote by $\Pi^{d}$ the set of polynomials in $d$ variables on $\mathbb{R}^{d}$ and by $\Pi_{n}^{d}$ the subset of polynomials of total degree at most $n$. We also denote by $\mathcal{P}_{n}^{d}$ the space of homogeneous polynomials of degree $n$ on $\mathbb{R}^{d}$ and we let $r_{n}^{d}=\operatorname{dim} \mathcal{P}_{n}^{d}$. It is well-known that

$$
\operatorname{dim} \Pi_{n}^{d}=\left(\begin{array}{c}
n+d \\
n
\end{array}\right) \quad \text { and } \quad r_{n}^{d}=\operatorname{dim} \mathcal{P}_{n}^{d}=\left(\begin{array}{c}
n+d-1 \\
d
\end{array}\right) .
$$

Weight functions. Throughout this paper a weight function on a compact region $\Omega$ is a nonnegative function on $\Omega$ whose integral over $\Omega$ is positive. We will take $\Omega=S^{d}, B^{d}$ or $\Sigma^{d}$ most of the time. On $B^{d}$ and $\Sigma^{d}$ we use $d \mathbf{x}$ (Lebesgue measure), on $S^{d}$ we use rotation invariant measure $d \omega$ (surface measure) on $S^{d}$.

Orthogonal polynomials on $B^{d}$ and $\Sigma^{d}$. Let $\Omega$ denote either $B^{d}$ or $\Sigma^{d}$. Let $W$ be a weight function on $\Omega$. It is known that for each $n \in \mathbb{N}_{0}$ the set of polynomials of degree $n$ that are orthogonal to all polynomials of lower degree forms a vector space $\mathcal{V}_{n}$ whose dimension is $r_{n}^{d}$. We denote by $\left\{P_{k}^{n}\right\}, 1 \leq k \leq r_{n}^{d}$ and $n \in \mathbb{N}_{0}$, one family of orthonormal polynomials with respect to $W$ on $\Omega$ that forms a basis of $\Pi_{n}^{d}$, where the superscript $n$ means that $P_{k}^{n} \in \Pi_{n}^{d}$. The orthonormality means that

$$
\int_{\Omega} P_{k}^{n}(\mathbf{x}) P_{j}^{m}(\mathbf{x}) W(\mathbf{x}) d \mathbf{x}=\delta_{j, k} \delta_{m, n}
$$

For each $n \in \mathbb{N}_{0}$, the polynomials $P_{k}^{n}, 1 \leq k \leq r_{n}^{d}$, form an orthonormal basis of $\mathcal{V}_{n}$. We note that there are many bases of $\mathcal{V}_{n}$; if $Q$ is an invertible matrix of size $r_{n}^{d}$, then the components of $Q \mathbb{P}_{n}$ form another basis of $\mathcal{V}_{n}$ which is orthonormal if $Q$ is an orthogonal matrix. For general results on orthogonal polynomials in several variables, including some of the recent development, we refer to the survey [23] and the references there.

Ordinary harmonics and h-harmonics. The ordinary harmonic polynomials on $\mathbb{R}^{d+1}$ are the homogeneous polynomials satisfying the Laplace equation $\Delta P=0$, where $\Delta=$ $\partial_{1}^{2}+\ldots+\partial_{d+1}^{2}$ on $\mathbb{R}^{d+1}$ and $\partial_{i}$ is the ordinary partial derivative with respect to the $i$-th coordinate. The spherical harmonics are the restriction of harmonic polynomials on $S^{d}$. They are orthogonal polynomials with respect to the surface measure $d \omega$ on $S^{d}$.

The theory of $h$-harmonics has been established recently by Dunkl (see [3-5]). For a nonzero vector $\mathbf{v} \in \mathbb{R}^{d+1}$ define the reflection $\sigma_{\mathbf{v}}$ by

$$
\mathbf{x} \sigma_{\mathbf{v}}:=\mathbf{x}-2(\mathbf{x} \cdot \mathbf{v}) \mathbf{v} /|\mathbf{v}|^{2}, \quad \mathbf{x} \in \mathbb{R}^{d+1}
$$


The $h$-harmonics are homogeneous orthogonal polynomials on $S^{d}$ with respect to $h_{\alpha}^{2} d \omega$, where the weight function $h_{\alpha}$ is defined by

$$
h_{\alpha}(\mathbf{x}):=\prod_{i=1}^{m}\left|\mathbf{x} \cdot \mathbf{v}_{i}\right|^{\alpha_{i}}, \quad \alpha_{i} \geq 0
$$

with $\alpha_{i}=\alpha_{j}$ whenever $\sigma_{i}$ is conjugate to $\sigma_{j}$ in the reflection group $G$ generated by the reflections $\left\{\sigma_{\mathbf{v}_{i}}: 1 \leq i \leq m\right\}$. The function $h_{\alpha}$ is a positively homogeneous $G$ invariant function. The key ingredient of the theory is a family of first-order differentialdifference operators, $\mathcal{D}_{i}$ (Dunkl's operators), which generates a commutative algebra ([5]). The $h$-Laplacian is defined by $\Delta_{h}=\mathcal{D}_{1}^{2}+\ldots+\mathcal{D}_{d+1}^{2}$, which plays the role of Laplacian in the theory of the ordinary harmonics. In particular, the $h$-harmonics are the homogeneous polynomials satisfying the equation $\Delta_{h} Y=0$. The $h$-spherical harmonics are the restriction of $h$-harmonics on the sphere.

Orthogonal polynomials on spheres for $S$-symmetric functions. A weight function $H$ defined on $\mathbb{R}^{d+1}$ is called $S$-symmetric if it is symmetric with respect to $y_{d+1}$ and centrally symmetric with respect to the variables $\mathbf{y}^{\prime}=\left(y_{1}, \ldots, y_{d}\right) ;$ i.e.,

$$
H\left(\mathbf{y}^{\prime}, y_{d+1}\right)=H\left(\mathbf{y}^{\prime},-y_{d+1}\right) \quad \text { and } \quad H\left(\mathbf{y}^{\prime}, y_{d+1}\right)=H\left(-\mathbf{y}^{\prime}, y_{d+1}\right)
$$

In particular, the weight functions of the form $H(\mathbf{y})=W\left(y_{1}^{2}, \ldots, y_{d+1}^{2}\right)$ are $S$-symmetric. It is proved in [25] that there is a unique decomposition

$$
\mathcal{P}_{n}^{d+1}=\bigoplus_{k=0}^{[n / 2]}|\mathbf{y}|^{2 k} \mathcal{H}_{n-2 k}(H)
$$

where $\mathcal{H}_{n}(H)$ is the subspace of homogeneous polynomials of degree $n$ which are orthogonal to polynomials of lower degree. If $H=h^{2}$, where $h$ is a reflection invariant weight function as in (2.2), then $\mathcal{H}_{n}(H)$ is the same as the space of $h$-harmonics on $\mathbb{R}^{d+1}$; in particular, when $H=1$, it is the same as the space of ordinary harmonics. It is also proved in [25] that an orthonormal basis of $\mathcal{H}_{n}^{d+1}$ can be constructed using orthogonal polynomials on $B^{d}$ as follows. Associated to the weight function $H$ we define two weight functions

$$
W^{(1)}(\mathbf{x})=H\left(\mathbf{x}, \sqrt{1-|\mathbf{x}|^{2}}\right) / \sqrt{1-|\mathbf{x}|^{2}} \text { and } \quad W^{(2)}(\mathbf{x})=H\left(\mathbf{x}, \sqrt{1-|\mathbf{x}|^{2}}\right) \sqrt{1-|\mathbf{x}|^{2}}
$$

on $B^{d}$. We denote by $\left\{P_{k}^{n}\right\}$ and $\left\{Q_{k}^{n}\right\}$ systems of orthonormal polynomials with respect to the weight functions $W^{(1)}$ and $W^{(2)}$, respectively, where we keep the convention that the superscript $n$ means that $P_{k}^{n}$ and $Q_{k}^{n}$ are polynomials in $\Pi_{n}^{d}$, and the subindex $k$ has the range $1 \leq k \leq r_{n}^{d}$. Keeping in mind the notation (2.1) we define

$$
Y_{k, n}^{(1)}(\mathbf{y})=r^{n} P_{k}^{n}(\mathbf{x}) \quad \text { and } \quad Y_{j, n}^{(2)}(\mathbf{y})=r^{n} x_{d+1} Q_{j}^{n-1}(\mathbf{x}),
$$


where $1 \leq k \leq r_{n}^{d}, 1 \leq j \leq r_{n-1}^{d}$ and we define $Y_{j, 0}^{(2)}(\mathbf{y})=0$. It is proved in [25] that these polynomials are homogeneous polynomials in $\mathbf{y}$ and they form an orthonormal basis for the space $\mathcal{H}_{n}(H)$.

The group $\mathbb{Z}_{2} \times \cdots \times \mathbb{Z}_{2}$. In the rest of this paper we will reserve the letter $G$ for the group $G=\mathbb{Z}_{2} \times \cdots \times \mathbb{Z}_{2}=\left(\mathbb{Z}_{2}\right)^{m}$. It is one of the simplest reflection groups. The elements of $G$ take the form $a=\left(\varepsilon_{1}, \ldots, \varepsilon_{m}\right)$, where $\varepsilon_{i}= \pm 1$. For a function $f$ defined on $\mathbb{R}^{m}$, the action of $a \in G$ on $f$ is defined by $R(a) f(\mathbf{x})=f(\mathbf{x} a), \mathbf{x} \in \mathbb{R}^{m}, a \in G$; we have $R(a) f(\mathbf{x})=f\left(\varepsilon_{1} x_{1}, \ldots, \varepsilon_{m} x_{m}\right)$. If $R(a) f=f$ for all $a \in G$, we say that $f$ is invariant under $G$. We take the convention that the size of $G$ agrees with the function it acts upon, so that we do not have to write $G=G_{m}$. We will take $m=d$ or $m=d+1$ in subsequent sections.

Basic Lemma. We let $d \omega=d \omega_{d}$ denote the surface measure on $S^{d}$, and the surface area

$$
\omega\left(S^{d}\right)=\omega_{d}\left(S^{d}\right)=\int_{S^{d}} d \omega_{d}=2 \pi^{(d+1) / 2} / \Gamma((d+1) / 2) .
$$

Let $H$ be defined on $\mathbb{R}^{d+1}$. Assume that $H$ is symmetric with respect to $x_{d+1}$; i.e., $H\left(\mathbf{x}, x_{d+1}\right)=H\left(\mathbf{x},-x_{d+1}\right)$, where $\mathbf{x} \in \mathbb{R}^{d}$. It is proved in [25] that for any continuous function $g$ defined on $S^{d}$, we have

$$
\begin{aligned}
& \int_{S^{d}} g(\mathbf{y}) H(\mathbf{y}) d \omega=\int_{B^{d}} {\left[g\left(\mathbf{x}, \sqrt{1-|\mathbf{x}|^{2}}\right)+g\left(\mathbf{x},-\sqrt{1-|\mathbf{x}|^{2}}\right)\right] } \\
& \times H\left(\mathbf{x}, \sqrt{1-|\mathbf{x}|^{2}}\right) d \mathbf{x} / \sqrt{1-|\mathbf{x}|^{2}}
\end{aligned}
$$

This formula connects the integral on $B^{d}$ to $S^{d}$ and it plays an essential role in [25]. The following establishes a relation between the integrations over the unit sphere and over the standard simplex.

Lemma 2.1. For any continuous function $f$ defined on $S^{d}$,

$$
\int_{S^{d}} f\left(y_{1}^{2}, \ldots, y_{d+1}^{2}\right) d \omega=2 \int_{\Sigma^{d}} f\left(u_{1}, \ldots, u_{d}, 1-|\mathbf{u}|_{1}\right) \frac{d \mathbf{u}}{\sqrt{u_{1} \ldots u_{d}\left(1-|\mathbf{u}|_{1}\right)}}
$$

Proof. It follows from (2.5) with $H=1$ and $g(\mathbf{y})=f\left(y_{1}^{2}, \ldots, y_{d+1}^{2}\right)$ that

$$
\int_{S^{d}} f\left(y_{1}^{2}, \ldots, y_{d+1}^{2}\right) d \omega=2 \int_{B^{d}} f\left(x_{1}^{2}, \ldots, x_{d}^{2}, 1-|\mathbf{x}|^{2}\right) \frac{d \mathbf{x}}{\sqrt{1-|\mathbf{x}|^{2}}} .
$$

Since the function $g\left(x_{1}^{2}, \ldots, x_{d}^{2}\right)$ is invariant under the action of $G$, we can write its integral over $B^{d}$ as $2^{d}$ times the integral over $B_{+}^{d}=\left\{\mathbf{x} \in B^{d}: x_{1} \geq 0, \ldots, x_{d} \geq 0\right\}$. Upon changing variables $u_{1}=x_{1}^{2}, \ldots, u_{d}=x_{d}^{2}$ in the integral over $B_{+}^{d}$, we obtain that

$$
\int_{B^{d}} g\left(x_{1}^{2}, \ldots, x_{d}^{2}\right) d \mathbf{x}=\int_{\Sigma^{d}} g\left(u_{1}, \ldots, u_{d}\right) \frac{d \mathbf{u}}{\sqrt{u_{1} \cdots u_{d}}}
$$


where we have changed the integral over $B_{+}^{d}$ back to the integral over $B^{d}$. Putting these two formulae together with $g(\mathbf{x})=f\left(\mathbf{x}, \sqrt{1-|\mathbf{x}|_{1}}\right) / \sqrt{1-|\mathbf{x}|_{1}}$ completes the proof.

In the case $d=2$ the formula (2.6) has appeared in [3, Lemma 1.6, p. 689]. We note, in particular, that the Lebesgue measure on $S^{d}$ is related to the weight function $1 / \sqrt{\left(u_{1} \ldots u_{d}\left(1-|\mathbf{u}|_{1}\right)\right.}$ over $\Sigma^{d}$.

\section{Orthogonal polynomials on Spheres AND ON Simplices}

In this section we discuss the relation between orthogonal polynomials on spheres and on simplices. We need some notations.

Definition 3.1. Let $H(\mathbf{y})=W\left(y_{1}^{2}, \ldots, y_{d+1}^{2}\right)$ be a weight function defined on $\mathbb{R}^{d+1}$. Associated to $H$ we define a weight function $W_{\Sigma}$ on the simplex $\Sigma^{d}$ by

$$
W_{\Sigma}(\mathbf{u})=2 W\left(u_{1}, \ldots, u_{d}, 1-|\mathbf{u}|_{1}\right) / \sqrt{u_{1} \cdots u_{d}\left(1-|\mathbf{u}|_{1}\right)}, \quad \mathbf{u} \in \Sigma^{d},
$$

and we normalize the weight function $W$ so that, recall the formula (2.6),

$$
\int_{S^{d}} W\left(y_{1}^{2}, \ldots, y_{d+1}^{2}\right) d \omega=\int_{\Sigma^{d}} W_{\Sigma}(\mathbf{u}) d \mathbf{u}=1 .
$$

It is evident that $H$ is $S$-symmetric on $\mathbb{R}^{d+1}$. Recall that the space of orthogonal homogeneous polynomials of degree $n$ associated to the weight function $H$ is denoted by $\mathcal{H}_{n}(H)$. If a polynomial $P$ in $\mathcal{H}_{n}(H)$ is invariant under $G$, then $P$ has to be of even degree, which means that $n$ is even. We define a subspace $\mathcal{H}_{2 n}^{G}$ of $\mathcal{H}_{2 n}(H)$ by

$$
\mathcal{H}_{2 n}^{G}=\left\{P \in \mathcal{H}_{2 n}(H) \mid P(\mathbf{x} a)=P(\mathbf{x}), \quad a \in G\right\} ;
$$

that is, $\mathcal{H}_{2 n}^{G}$ contains polynomials in $\mathcal{H}_{2 n}(H)$ that are invariant under the action of $G$. Similarly, we define $\mathcal{P}_{2 n}^{G}$ to be the subspace of $\mathcal{P}_{2 n}^{d+1}$ containing all invariant homogeneous polynomials on $\mathbb{R}^{d+1}$.

Let $R_{k}^{n}$ denote a basis of orthonormal polynomials of degree $n$ with respect to the weight function $W_{\Sigma}$ on $\Sigma^{d}$, where we keep the convention that the superscript $n$ means that $R_{k}^{n}$ are polynomials in $\Pi_{n}^{d}$, and the subindex $k$ has the range $1 \leq k \leq r_{n}^{d}$. Keep in mind the notation (2.1). We define

$$
S_{k}^{2 n}(\mathbf{y})=r^{2 n} R_{k}^{n}\left(x_{1}^{2}, \ldots, x_{d}^{2}\right), \quad 1 \leq k \leq r_{n}^{d} .
$$

These polynomials are in fact homogeneous polynomials in $\mathbf{y} \in \mathbb{R}^{d+1}$.

Theorem 3.2. Let $W$ be a weight function on $\mathbb{R}^{d+1}$ defined as in Definition 3.1. Then the functions $S_{k}^{2 n}$ defined in (3.3) are homogeneous polynomials of degree $2 n$ on $\mathbb{R}^{d+1}$, and the set $\left\{S_{k}^{2 n}: 1 \leq k \leq r_{n}^{d}\right\}$ forms an orthonormal basis for $\mathcal{H}_{2 n}^{G}$. Moreover,

$$
\mathcal{P}_{2 n}^{G}=\bigoplus_{k=0}^{n}|\mathbf{y}|^{2 k} \mathcal{H}_{2 n-2 k}^{G}
$$


that is, if $P \in \mathcal{P}_{n}^{G}$, then there is a unique decomposition

$$
P(\mathbf{y})=\sum_{k=0}^{n}|\mathbf{y}|^{2 n-2 k} P_{2 k}(\mathbf{y}), \quad P_{2 k} \in \mathcal{H}_{2 k}^{G}
$$

Proof. Recall the notation (2.1); we see that $S_{k}^{2 n}$ takes the form

$$
S_{k}^{2 n}(\mathbf{y})=r^{2 n} R_{k}^{n}\left(x_{1}^{2}, \ldots, x_{d}^{2}\right)=r^{2 n} \sum_{|\beta|_{1} \leq n} a_{\beta} x_{1}^{2 \beta_{1}} \cdots x_{d}^{2 \beta_{d}}
$$

where $a_{\beta}$ are constants. Since $y_{i}=r x_{i}$, it is not hard to see that $S_{k}^{2 n}$ is a homogeneous polynomial of degree $2 n$ in the variables $\mathbf{y} \in \mathbb{R}^{d+1}$. To show $S_{k}^{2 n} \in \mathcal{H}_{n}(H)$, we need to show that it is orthogonal to polynomials of degree less than $2 n$ with respect to $H(\mathbf{y}) d \omega$ on $S^{d}$. We consider $P_{\beta}(\mathbf{y})=\mathbf{y}^{\beta}$ for $\beta \in \mathbb{N}^{d+1}$ and $|\beta|_{1} \leq 2 n-1$. If one of the components of $\beta=\left(\beta_{1}, \ldots, \beta_{d+1}\right)$ is odd, say $\beta_{1}$ is odd, then we conclude by symmetry of $x_{1} \mapsto-x_{1}$ that

$$
\int_{S^{d}} S_{k}^{2 n}(\mathbf{y}) P_{\beta}(\mathbf{y}) H(\mathbf{y}) d \omega=\int_{S^{d}} R_{k}^{n}\left(y_{1}^{2}, \ldots, y_{d}^{2}\right) \mathbf{y}^{\beta} W\left(y_{1}^{2}, \ldots, y_{d+1}^{2}\right) d \omega=0
$$

Hence, we only need to consider the case when all components of $\beta$ are even; in such a case we write $\beta=2 \gamma$ for $\gamma \in \mathbb{N}^{d+1}$ and $|\gamma|_{1} \leq n-1$. Then the basic formula (2.6) shows that

$$
\int_{S^{d}} S_{k}^{2 n}(\mathbf{y}) \mathbf{y}^{2 \gamma} H(\mathbf{y}) d \omega=\int_{\Sigma^{d}} R_{k}^{n}(\mathbf{x}) x_{1}^{\gamma_{1}} \cdots x_{d}^{\gamma_{d}}\left(1-x_{1}-\cdots-x_{d}\right)^{\gamma_{d+1}} W_{\Sigma}(\mathbf{x}) d \mathbf{x}=0
$$

by the orthogonality of $R_{k}^{n}$ to polynomials of lower degrees. Since it is evident from (3.3) that $S_{k}^{2 n}$ are invariant under $G$, we have shown that $S_{k}^{2 n}$ are elements of $\mathcal{H}_{2 n}^{G}$. Moreover, if we replace $\mathbf{y}^{2 \gamma}$ by $S_{j}^{2 n}(\mathbf{y})$ in the above formula, then it follows from the orthonormality of $R_{k}^{n}$ that $\left\{S_{k}^{2 n}: 1 \leq k \leq r_{n}^{d}\right\}$ forms an orthonormal set.

Let $P$ be a homogeneous polynomial on $\mathbb{R}^{d+1}$ that is invariant under $G$. Then $P$ is necessarily of even degree, say degree $2 n$, which is of the form $P(\mathbf{y})=p\left(y_{1}^{2}, \ldots, y_{d+1}^{2}\right)$, where $p$ is homogeneous of degree $n$. Using $x_{d+1}^{2}=1-x_{1}^{2}-\ldots x_{d}^{2}$ whenever possible, we can write $P$ further as

$$
P(\mathbf{y})=r^{2 n} q\left(x_{1}^{2}, \ldots, x_{d}^{2}\right), \quad q \in \Pi_{n}^{d},
$$

where $q$ is a polynomial of degree $n$, not homogeneous in general. Since $\left\{R_{j}^{k}, 1 \leq j \leq\right.$ $\left.r_{n}^{d}, 0 \leq k \leq n\right\}$ forms a basis for all polynomials of degree $n$, we can write $q$ in terms of $R_{j}^{k}$. Therefore, we have the unique expansion

$$
P(\mathbf{y})=r^{2 n} \sum_{k=0}^{n} \sum_{j=1}^{r_{k}^{d}} b_{j, k} R_{j}^{k}\left(x_{1}^{2}, \ldots, x_{d}^{2}\right)=\sum_{k=0}^{n} \sum_{j=1}^{r_{k}^{d}} b_{j, k} r^{2 n-2 k} S_{j}^{2 k}(\mathbf{y})
$$


In particular, if $P \in \mathcal{H}_{2 n}^{G}$, then it follows from the orthogonality of $P$ to polynomials of lower degree that $P(\mathbf{y})=\sum_{j} a_{j} S_{j}^{2 n}(\mathbf{y})$. This shows that $\left\{S_{j}^{n}, 1 \leq j \leq r_{n}^{d}\right\}$ forms an orthonormal basis of $\mathcal{H}_{2 n}^{G}$, and the unique decomposition (3.4) holds.

As a special case of the theorem, a basis of orthonormal polynomials associated to the weight function $W_{\Sigma}(\mathbf{u})=\left(u_{1} \cdots u_{d}\left(1-|\mathbf{u}|_{1}\right)\right)^{-1 / 2}$ yields, by (3.3), an orthonormal basis for spherical harmonics that are invariant under $G$.

Since $\left\{S_{j}^{n}\right\}$ forms a basis of $\mathcal{H}_{2 n}^{G}$, it follows that the dimension of $\mathcal{H}_{2 n}^{G}$ is $r_{n}^{d}$. We formulate this as a corollary.

Corollary 3.3. Let $W$ be a weight function on $\mathbb{R}^{d+1}$ defined as in Definition 3.1. Then

$$
\operatorname{dim} \mathcal{H}_{2 n}^{G}=\left(\begin{array}{c}
n+d-1 \\
n
\end{array}\right) \quad \text { and } \quad \operatorname{dim} \mathcal{P}_{2 n}^{G}=\left(\begin{array}{c}
n+d \\
n
\end{array}\right) .
$$

For the $h$-harmonics, the decomposition of $\mathcal{P}_{2 n}^{G}$ in terms of $\mathcal{H}_{2 n}^{G}$ was established in [4] using the differential-difference operators. The importance of the above theorem lies in the relation between orthogonal polynomials on the sphere $S^{d}$ and on the simplex $\Sigma^{d}$. In view of the result in [25], we can also establish a relation between orthogonal polynomials on the simplex $\Sigma^{d}$ and on the ball $B^{d}$.

Let $U$ be a weight function defined on $\mathbb{R}^{d}$. We define a weight function $U_{\Sigma}$ on $\Sigma^{d}$ by

$$
U_{\Sigma}(\mathbf{u})=U\left(u_{1}, \ldots, u_{d}\right) / \sqrt{u_{1} \cdots u_{d}}, \quad \mathbf{u} \in \Sigma^{d} .
$$

We denote the space of orthogonal polynomials of degree $n$ with respect to the weight function $U\left(x_{1}^{2}, \ldots, x_{d}^{2}\right)$ on $B^{d}$ by $\mathcal{V}_{n}\left(B^{d}\right)$. If $P \in \mathcal{V}_{n}\left(B^{d}\right)$ is invariant under $G$, then it must be of even degree. We define a subspace $\mathcal{V}_{2 n}^{G}\left(B^{d}\right)$ of $\mathcal{V}_{2 n}\left(B^{d}\right)$ by

$$
\mathcal{V}_{2 n}^{G}\left(B^{d}\right)=\left\{P \in \mathcal{V}_{2 n}\left(B^{d}\right) \mid P(\mathbf{x} a)=P(\mathbf{x}), \quad a \in G\right\} .
$$

That is, $\mathcal{V}_{2 n}\left(B^{d}\right)$ contains orthogonal polynomials in $\mathcal{V}_{2 n}\left(B^{d}\right)$ that are invariant under G.

Theorem 3.4. Let $U$ be a weight function defined on $\mathbb{R}^{d}$. Let $R_{k}^{n}$ be orthonormal polynomials with respect to the weight function $U_{\Sigma}$. Then the polynomials $T_{k}^{2 n}(\mathbf{x})=$ $R_{k}^{n}\left(x_{1}^{2}, \ldots, x_{d}^{2}\right)$ are orthogonal with respect to the weight function $U\left(x_{1}^{2}, \ldots, x_{d}^{2}\right)$ on $B^{d}$ and $\left\{T_{k}^{2 n}, 1 \leq k \leq r_{n}^{d}\right\}$ forms an orthonormal basis for $\mathcal{V}_{2 n}^{G}\left(B^{d}\right)$. In particular, it follows that $\operatorname{dim} \mathcal{V}_{2 n}^{G}\left(B^{d}\right)=r_{n}^{d}$.

The theorem follows as a corollary of Theorem 3.2 and the results in [25]. It can also be proved following a similar line as in the proof of Theorem 3.2, using the formula (2.7) instead of (2.6); we leave the details to the reader.

These two theorems point out a rather basic relation between orthogonal polynomials on the simplex $\Sigma^{d}$ and those on the sphere $S^{d}$ and the ball $B^{d}$. It is rather remarkable that, except for the case discussed in [3], they do not seem to have appeared in the literature. Let us illustrate them by an example, in which we look at the classical orthogonal polynomials. 
Example 3.5. Let us consider the weight functions $H(\mathbf{y})=H_{\alpha}\left|y_{1}\right|^{2 \alpha_{1}}\left|y_{2}\right|^{2 \alpha_{2}}\left|y_{3}\right|^{2 \alpha_{3}}$ on $S^{2}$, where $H_{\alpha}$ is a normalization constant so that $H$ is normalized as in Definition 3.1. The corresponding weight function $W_{\Sigma}$ on the simplex $\Sigma^{d}$ is given by

$$
W_{\Sigma}^{\alpha}(\mathbf{x})=2 H_{\alpha} x_{1}^{\alpha_{1}-1 / 2} x_{2}^{\alpha_{2}-1 / 2}\left(1-x_{1}-x_{2}\right)^{\alpha_{3}-1 / 2} .
$$

In an effort to understand Dunkl's theory of $h$-harmonics, in [24] we study the orthogonal polynomials on $S^{d}$ associated to $H(\mathbf{y})=H_{\alpha} y_{1}^{\alpha_{1}} \cdots y_{d+1}^{\alpha_{d+1}}, d \geq 2$, in detail. In particular, an orthonormal basis of $h$-harmonics is given in terms of the orthonormal polynomials $D_{n}^{(\lambda, \mu)}$ of one variable with respect to the measure $\left(1-t^{2}\right)^{\lambda}|t|^{2 \mu}$ on $[-1,1]$, which in turn can be written in terms of Jacobi polynomials. For $d=2$, using the spherical coordinates

$$
y_{1}=r \cos \theta_{1}, \quad y_{2}=r \sin \theta_{1} \cos \theta_{2}, \quad y_{3}=r \sin \theta_{1} \sin \theta_{2}, \quad r=|\mathbf{y}|
$$

some of the $h$-harmonics of degree $n\left(Y_{k}^{n,(1)}\right.$ in the notation of [24]) are given by

$$
Y_{k}^{n}(\mathbf{y})=A_{k}^{n} r^{n} D_{n-k}^{\left(k+\alpha_{2}+\alpha_{3}+1 / 2, \alpha_{1}\right)}\left(\cos \theta_{1}\right)\left(\sin \theta_{1}\right)^{k} D_{k}^{\left(\alpha_{3}, \alpha_{2}\right)}\left(\cos \theta_{2}\right)
$$

where $0 \leq k \leq n$ and $A_{k}^{n}$ are normalization constants. (It should be mentioned that the spherical coordinates adopted above are in the reverse order of the spherical coordinates used in [24].) On the other hand, an orthonormal basis for $W_{\Sigma}$ can be given in terms of the Jacobi polynomials $P_{n}^{(\alpha, \beta)}$ by (cf. [10])

$$
R_{k}^{n}\left(x_{1}, x_{2}\right)=B_{k}^{n} P_{n-k}^{\left(2 k+\alpha_{2}+\alpha_{3}, \alpha_{1}-1 / 2\right)}\left(2 x_{1}-1\right)\left(1-x_{1}\right)^{k} P_{k}^{\left(\alpha_{3}-1 / 2, \alpha_{2}-1 / 2\right)}\left(2 \frac{x_{2}}{1-x_{1}}-1\right)
$$

where $0 \leq k \leq n, n \geq 0$, and $B_{k}^{n}$ are normalization constants. According to Theorem 3.2 , the polynomials

$$
S_{k}^{2 n}(\mathbf{y})=r^{2 n} R_{k}^{n}\left(x_{1}^{2}, x_{2}^{2}\right), \quad \mathbf{y}=\left(y_{1}, y_{2}, y_{3}\right)=r\left(x_{1}, x_{2}, x_{3}\right)
$$

are $h$-harmonics with respect to $H$. In fact, using the spherical coordinates and the formula $D_{2 n}^{(\lambda, \mu)}(\cos \theta)=$ const $P_{n}^{(\lambda-1 / 2, \mu-1 / 2)}(\cos 2 \theta)([24])$, we see from the above formulae for $Y_{k}^{n}$ and $R_{k}^{n}$ that, up to a constant, the polynomials $S_{k}^{2 n}(\mathbf{y})$ and $Y_{2 k}^{2 n}(\mathbf{y})$ are identical. In particular, this shows that $Y_{2 k}^{2 n}(\mathbf{y})$ forms a basis for $h$-harmonics invariant under $G$.

Moreover, by Theorem 3.4, the polynomials $T_{k}^{n}\left(x_{1}, x_{2}\right)=R_{k}^{n}\left(x_{1}^{2}, x_{2}^{2}\right)$ are orthogonal polynomials on $B^{2}$ with respect to the weight function

$$
U\left(x_{1}, x_{2}\right)=2 H_{\alpha}\left|x_{1}\right|^{2 \alpha_{1}}\left|x_{2}\right|^{2 \alpha_{2}}\left(1-x_{1}^{2}-x_{2}^{2}\right)^{2 \alpha_{3}-1 / 2} \text {. }
$$

If $\alpha_{1}=\alpha_{2}=0$, we can use the quadratic transform $P_{2 k}^{(\lambda, \lambda)}(t)=$ const $P_{k}^{(\lambda,-1 / 2)}\left(2 t^{2}-1\right)$ $[18$, p. $59,(4.1 .5)]$ to conclude that

$$
T_{k}^{n}\left(x_{1}, x_{2}\right)=\operatorname{const} P_{2 n-2 k}^{\left(2 k+\alpha_{3}, 2 k+\alpha_{3}\right)}\left(x_{1}\right)\left(1-x_{1}^{2}\right)^{k} P_{2 k}^{\left(\alpha_{3}-1 / 2, \alpha_{3}-1 / 2\right)}\left(x_{2} / \sqrt{1-x_{1}^{2}}\right) .
$$


Apart from the constant, these polynomials with $\alpha=\alpha_{3}-1 / 2$ are exactly the same as ${ }_{2} P_{2 n, 2 k}^{\alpha}$ given in [10, formula (3.8), p. 449], where the polynomials ${ }_{2} P_{n, k}^{\alpha}$ are given as an orthogonal basis for the weight function $\left(1-x_{1}^{2}-x_{2}^{2}\right)^{\alpha-1 / 2}$.

The case $\alpha_{1}=\alpha_{2}=\alpha_{3}=0$ in the above example is of particular interest, since $h$-harmonics reduce to the ordinary harmonics when $\alpha=0$. Another interesting case is $\alpha_{1}=\alpha_{2}=\alpha_{3}$; Dunkl [3] studied the $h$-harmonics that are invariant under the symmetric group of order three in this case. Among other things, an orthogonal basis invariant under the permutations of $x_{1}, x_{2}$, and $1-x_{1}-x_{2}$ is given, which leads to $h$-harmonics invariant under the octahedral group.

\section{Cubature formula on spheres AND ON Simplices}

In this section we discuss the connection between cubature formulae on spheres and on simplices. For a given integral $\mathcal{L}(f):=\int f d \mu$, where $d \mu$ is a nonnegative measure with support set on $\Sigma^{d}$ or $B^{d}$, a cubature formula of degree $M$ is a linear functional

$$
\mathcal{I}_{M}(f)=\sum_{k=1}^{N} \lambda_{k} f\left(\mathbf{x}_{k}\right), \quad \lambda_{k} \in \mathbb{R}, \quad \mathbf{x}_{k} \in \mathbb{R}^{d},
$$

defined on $\Pi^{d}$, such that $\mathcal{L}(f)=\mathcal{I}_{M}(f)$ whenever $f \in \Pi_{M}^{d}$, and $\mathcal{L}\left(f^{*}\right) \neq \mathcal{I}_{M}\left(f^{*}\right)$ for at least one $f^{*} \in \Pi_{M+1}^{d}$. When the measure is supported on $S^{d}$, we need to replace $\Pi_{M}^{d}$ by $\Pi_{M}^{d+1}$ in the above formulation and require $\mathbf{x}_{k} \in S^{d}$. The points $\mathbf{x}_{1}, \ldots, \mathbf{x}_{N}$ in the formula are called nodes and the numbers $\lambda_{1}, \ldots, \lambda_{N}$ are called weights. Such a formula is called minimal, if $N$, the number of nodes, is minimal among all cubature formulae of degree $M$.

We are interested in the cubature formulae that are invariant under the action of the group $G$. A linear functional $\mathcal{I}(f)$ is called invariant under $G$ if $\mathcal{I}(R(a) f)=\mathcal{I}(f)$ for all $a \in G$. In order to write down an invariant cubature formula explicitly, we need more notation. For $\mathbf{u} \in \mathbb{R}^{m}, m=d$ or $d+1$, we denote its $G$-orbit by $G(\mathbf{u})$, which is defined by $G(\mathbf{u})=\{\mathbf{u} a \mid a \in G\}$; we also denote by $|G(\mathbf{u})|$ the number of distinct elements in $G(\mathbf{u})$. A cubature formula is invariant under $G$ if the set of its nodes is a union of $G$-orbits and the nodes belonging to the same $G$-orbit have the same weight. In the case we are considering, the invariant cubature formula, denoted by $I_{M}^{G}(f)$, takes the form

$$
\mathcal{I}_{M}^{G}(f)=\sum_{k=1}^{N} \lambda_{k} \sum_{a \in G} f\left(\mathbf{u}_{i} a\right) /\left|G\left(\mathbf{u}_{i}\right)\right|=\sum_{k=1}^{N} \lambda_{k} \sum_{\varepsilon \in\{-1,1\}^{m}} f\left(\varepsilon_{1} u_{i, 1}, \ldots \varepsilon_{m} u_{i, m}\right) /\left|G\left(\mathbf{u}_{i}\right)\right|,
$$

where the summation $\sum_{a \in G} f\left(\mathbf{u}_{i} a\right)$ is understood as taken over all distinguished elements of $G\left(\mathbf{u}_{i}\right)$ as explained in the second equality. We note that $|G(\mathbf{u})|=2^{k}$, where $k$ is the number of non-zero elements of $\mathbf{u}$ in this case.

The main result in this section establishes a correspondence between cubature formulae on the simplex $\Sigma^{d}$ and a $\mathbb{Z}_{2} \times \cdots \times \mathbb{Z}_{2}$ invariant cubature formulae on the sphere $S^{d}$. Because of their applications in numerical integration and in areas ranging from coding 
theory to isometric embeddings between classical Banach spaces, cubature formulae on the sphere have been under intense study for years. One of the important approaches in the construction of cubature formulae is initiated by Sobolev [16], which deals with the invariant cubature formula under a finite group. The fundamental result of Sobolev states that a cubature formula invariant under a group is exact for all polynomials in a subspace $\mathcal{P}$ if, and only if, it is exact for all polynomials in $\mathcal{P}$ that are invariant under the same group. Since this result helps to reduce the number of polynomials that need to be evaluated by a great deal, it has been used to construct a number of cubature formulae on the unit sphere $S^{2}$ (cf. $[6,11,15,17]$ and the references there). In the case we are considering, the results in the previous section shows that the invariant polynomials on $S^{d}$ can be identified with the polynomials on the simplex $\Sigma^{d}$ under a proper change of variables. Therefore, Sobolev's result suggests that the $\mathbb{Z}_{2} \times \cdots \times \mathbb{Z}_{2}$ invariant cubature formulae are related to the cubature formulae on the simplex. Indeed, we have the following result which does not seem to have been noticed before.

Theorem 4.1. Let $W_{\Sigma}$ be a weight function on $\Sigma^{d}$ as defined in Definition 3.1. Suppose that there is a cubature formula of degree $M$ on $\Sigma^{d}$ giving by

$$
\int_{\Sigma^{d}} f(\mathbf{u}) W_{\Sigma}(\mathbf{u}) d \mathbf{u}=\sum_{i=1}^{N} \lambda_{i} f\left(\mathbf{u}_{i}\right), \quad f \in \Pi_{M}^{d}
$$

whose $N$ nodes lie on the simplex $\Sigma^{d}$; that is $\mathbf{u}_{i}=\left(u_{i, 1}, \ldots, u_{i, d}\right)$ and $u_{i} \geq 0,1-|\mathbf{u}|_{1} \geq 0$. Then there is a cubature formula of degree $2 M+1$ on the unit sphere $S^{d}$,

$$
\int_{S^{d}} g(\mathbf{y}) W\left(y_{1}^{2}, \ldots, y_{d+1}^{2}\right) d \omega=\sum_{i=1}^{N} \lambda_{i} \sum_{a \in G} g\left(\mathbf{v}_{i} a\right) /\left|G\left(\mathbf{v}_{i}\right)\right|, \quad g \in \Pi_{2 M+1}^{d+1}
$$

where the nodes $\mathbf{v}_{i}=\left(v_{i, 1}, \ldots, v_{i, d+1}\right) \in S^{d}$ are defined in terms of $\mathbf{u}_{i}$ by

$$
\mathbf{v}_{i}=\left(v_{i, 1}, \ldots, v_{i, d+1}\right)=\left(\sqrt{u_{i, 1}}, \ldots, \sqrt{u_{i, d}}, \sqrt{1-\left|\mathbf{u}_{i}\right|_{1}}\right)
$$

On the other hand, if there exists a cubature formula of degree $2 M+1$ on $S^{d}$ in the form of (4.2), then there is a cubature formula of degree $M$ on the simplex $\Sigma^{d}$ in the form of (4.1) whose nodes $\mathbf{u}_{i} \in \Sigma^{d}$ are defined by

$$
\mathbf{u}_{i}=\left(u_{i, 1}, \ldots, u_{i, d}\right)=\left(v_{i, 1}^{2}, \ldots, v_{i, d}^{2}\right) .
$$

Proof. Assuming (4.1), we prove (4.2) for the polynomials $g_{\beta}(\mathbf{y})=\mathbf{y}^{\beta}, \beta \in \mathbb{N}^{d+1}$ and $|\beta|_{1} \leq 2 M+1$. If one of the components of $\beta=\left(\beta_{1}, \ldots, \beta_{d}\right)$ is odd, say $\beta_{1}$ is odd, then the integral in the left hand side of (4.2) is zero by symmetry. Moreover, let $a_{0}=$ $(-1,1, \ldots 1) \in G$; we have that

$$
\sum_{a \in G} g_{\beta}\left(\mathbf{v}_{i} a\right)=\sum_{a \in G} g_{\beta}\left(\mathbf{v}_{i} a_{0} a_{0}^{-1} a\right)=\sum_{b \in G} g_{\beta}\left(\mathbf{v}_{i} a_{0} b\right)=-\sum_{b \in G} g_{\beta}\left(\mathbf{v}_{i} b\right),
$$


which implies that the right hand side of (4.2) is also zero. Hence, we only need to verify (4.2) for $g_{2 \gamma}(\mathbf{y})=\mathbf{y}^{2 \gamma},|\gamma|_{1} \leq M$. Since $g_{2 \gamma}$ is clearly invariant under $G$, the right hand side of (4.2) with $g=g_{2 \gamma}$ becomes

$$
\sum_{i=1}^{N} \lambda_{i} \sum_{a \in G} g_{2 \gamma}\left(\mathbf{v}_{i} a\right) /\left|G\left(\mathbf{v}_{i}\right)\right|=\sum_{i=1}^{N} \lambda_{i} g_{2 \gamma}\left(\mathbf{v}_{i}\right) .
$$

Hence, taking into consideration (4.3), it follows from the basic formula (2.6) that (4.2) with $g=g_{2 \gamma}$ is equivalent to (4.1) with $f(\mathbf{u})=u_{1}^{\gamma_{1}} \cdots u_{d}^{\gamma_{d}}\left(1-|\mathbf{u}|_{1}\right)^{\gamma_{d+1}}$, which holds true since $|\gamma|_{1} \leq M$.

On the other hand, assuming (4.2), to prove (4.1) we only have to restrict (4.2) to invariant polynomials of the form $g(\mathbf{y})=f\left(y_{1}^{2}, \ldots, y_{d}^{2}\right), \mathbf{y} \in \mathbb{R}^{d+1}$, and use the basic formula (2.6).

Although both the statement and the proof of this theorem are simple, its importance is apparent. It allows us to transform back and forth between cubature formulae on $\Sigma^{d}$ and $\mathbb{Z}_{2} \times \cdots \times \mathbb{Z}_{2}$ invariant cubature formulae on $S^{d}$ for a large class of weight functions. Before we go any further, let us determine the number of nodes in the cubature formula (4.2).

Evidently, the number of nodes of (4.2) depends on how many nodes of (4.1) are on the boundary of $\Sigma^{d}$. Recall that $\Sigma^{d}$ is defined by $d+1$ inequalities: $u_{1} \geq 0, \ldots, u_{d} \geq 0$ and $1-|\mathbf{u}|_{1} \geq 0$ for $\mathbf{u} \in \mathbb{R}^{d}$. A $k$-dimensional face of $\Sigma^{d}, 0 \leq k \leq d$, contains elements of $\Sigma^{d}$ for which exactly $d-k$ inequalities become equalities. In particular, if $k=d$, then none of the inequalities becomes equality, so that the (unique) $d$-dimensional face of $\Sigma^{d}$ is the interior of $\Sigma^{d}$. We also note that a 0 -dimensional face is one of the vertices of the simplex $\Sigma^{d}$. For $0 \leq k \leq d$, we define

$$
N_{k}=\text { number of nodes in (4.1) which lie on } k \text {-dimensional faces. }
$$

Then the number of nodes of (4.1) is equal to $N_{0}+N_{1}+\ldots N_{d}$. We have the following corollary.

Corollary 4.2. Let $N_{M}\left(\Sigma^{d}\right)$ and $N_{2 M+1}\left(S^{d}\right)$ denote the number of nodes of the formula (4.1) and the formula (4.2), respectively. Then the following relation holds

$$
N_{M}\left(\Sigma^{d}\right)=N_{0}+N_{1}+\ldots N_{d} \quad \Longleftrightarrow \quad N_{2 M+1}\left(S^{d}\right)=2^{d+1} N_{d}+\ldots+4 N_{1}+2 N_{0} .
$$

Proof. If a node $\mathbf{u}_{i}$ of (4.1) lies on a $k$-dimensional face of $\Sigma^{d}$, then it follows from the definition that the corresponding node $\mathbf{v}_{i}$ in (4.3) has exactly $k+1$ nonzero elements, which implies that $\left|G\left(\mathbf{u}_{i}\right)\right|=2^{k+1}$. Moreover, if $\mathbf{v}_{i}$ is a node of a cubature formula of the form (4.2) which contains exactly $k+1$ nonzero elements, then $\mathbf{u}_{i}$ in (4.4) lies on a $k$-dimensional face of $\Sigma^{d}$.

There are lower bounds on the number of nodes of cubature formulae, which are used to test whether a given cubature formula is minimal. The lower bound for cubature formulae on $\Sigma^{d}$ is given by

$$
N_{M}\left(\Sigma^{d}\right) \geq\left(\begin{array}{c}
n+d \\
n
\end{array}\right):=N_{M}^{\min }\left(\Sigma^{d}\right), \quad M=2 n \quad \text { or } \quad M=2 n+1 .
$$


This lower bound is well-known (cf. [17]) and it holds for all cubature formulae with respect to weight functions on $\Sigma^{d}$ or on any other domain that has a positive measure. For cubature formulae of odd degree with respect to the weight function $W_{\Sigma}^{\alpha}$ on $\Sigma^{2}$ in (3.6), there is an improved lower bound ([2])

$$
N_{2 n+1}\left(\Sigma^{2}\right) \geq\left(\begin{array}{c}
n+2 \\
n
\end{array}\right)+\left[\frac{n+1}{2}\right]:=N_{2 n+1}^{\min }\left(\Sigma^{2}\right),
$$

which is the same as the lower bound for cubature formulae of degree $2 n+1$ with respect to a centrally symmetric weight function, such as $\left(1-|\mathbf{x}|^{2}\right)^{\alpha}$ on $B^{2}$. This bound follows from a general result of Möller ([14]), but the verification $([2,21])$ uses explicit formulae for the orthogonal polynomials with respect to $W_{\Sigma}^{\alpha}$. It is not known whether the lower bound (4.7) holds for other weight functions on $\Sigma^{2}$. For cubature formulae on $S^{d}$ we only give the lower bound for odd degree, since the cubature formula (4.2) is of degree $2 M+1$. We have

$$
N_{2 M+1}\left(S^{d}\right) \geq 2\left(\begin{array}{c}
M+d \\
M
\end{array}\right):=N_{2 M+1}^{\min }\left(S^{d}\right),
$$

(cf. $[14,15]$ ) which holds for all centrally symmetric weight functions on $S^{d}$. We note that for large $M$, the order of both $N_{M}^{\min }\left(\Sigma^{d}\right)$ and $N_{2 M+1}^{\min }\left(S^{d}\right)$ is $\mathcal{O}\left(M^{d}\right)$.

The following corollary states that if the cubature formula (4.1) is close to a minimal formula for $M$ relatively large, then so is the $\mathbb{Z}_{2} \times \cdots \times \mathbb{Z}_{2}$ invariant cubature formula (4.2).

Corollary 4.3. Let the notation be the same as in Corollary 4.2. Then

$$
N_{M}\left(\Sigma^{d}\right)=N_{M}^{\min }\left(\Sigma^{d}\right)+\mathcal{O}\left(M^{d-1}\right) \quad \Longleftrightarrow \quad N_{2 M+1}\left(S^{d}\right)=N_{2 M+1}^{\min }\left(S^{d}\right)+\mathcal{O}\left(M^{d-1}\right) .
$$

Proof. This follows easily from the following asymptotic formula

$$
\left(\begin{array}{c}
n+\lambda \\
n
\end{array}\right)=\frac{\Gamma(n+\lambda+1)}{\Gamma(\lambda+1) \Gamma(n+1)}=\frac{n^{\lambda}}{\Gamma(\lambda+1)}\left(1+\mathcal{O}\left(n^{-1}\right)\right), \quad \lambda>0,
$$

and the formulae (4.6) and (4.8).

It is worth mentioning that an attractive feature of the correspondence in Theorem 4.1 is that the degree of the $\mathbb{Z}_{2} \times \cdots \times \mathbb{Z}_{2}$ invariant cubature formula (4.2) is more than twice the degree of the cubature formula (4.1) on $\Sigma^{d}$. Since it is easier to construct cubature formulae of lower degree, the theorem offers an effective method to construct cubature formulae on the sphere. However, most of the existing cubature formulae on $\Sigma^{d}$ are constructed for the Lebesgue measure $d \mathbf{x}$ (see the survey [12]), which corresponds to the measure $\left|y_{1} \cdots y_{d+1}\right| d \omega$ on $S^{d}$; and there are few cubature formulae known for the weight function $\left(u_{1} \cdots u_{d}\left(1-u_{1}-\cdots-u_{d}\right)\right)^{1 / 2}$ on $\Sigma^{d}$, which corresponds to the surface measure $d \omega$. On the other hand, most of the existing cubature formulae on $S^{d}$ are constructed for the surface measure $d \omega$, from which we may obtain cubature formula on $\Sigma^{d}$ with respect 
to the measure $\left(u_{1} \cdots u_{d}\left(1-u_{1}-\cdots-u_{d}\right)\right)^{1 / 2}$. Hence, Theorem 4.1 will also help us to obtain new cubature formulae on $\Sigma^{d}$. We indicate the power of the method by the following example.

Example 4.4. The following is a $\mathbb{Z}_{2} \times \mathbb{Z}_{2} \times \mathbb{Z}_{2}$ invariant cubature formula of degree 5 on the unit sphere $S^{2}$ with 12 nodes ([1] and [17, p. 296, formula $\left.U_{3}: 5-1\right]$ ),

$$
\int_{S^{2}} f\left(y_{1}, y_{2}, y_{3}\right) d \omega=\frac{\pi}{3} \sum[f( \pm r, \pm s, 0)+f(0, \pm r, \pm s)+f( \pm s, 0, \pm r)],
$$

where the sum is taken over all possible choices of $( \pm 1, \pm 1)$, and $r, s$ are given by

$$
r^{2}=(5+\sqrt{5}) / 10 \text { and } s^{2}=(5-\sqrt{5}) / 10 .
$$

It is a minimal cubature formula on $S^{2}$ by (4.8). We note that $r^{2}+s^{2}=1$. Using Theorem 4.1, we can transform it to a cubature formula of degree 2 on $\Sigma^{2}$,

$$
\int_{\Sigma^{2}} f\left(x_{1}, x_{2}\right) \frac{d x_{1} d x_{2}}{\sqrt{x_{1} x_{2}\left(1-x_{1}-x_{2}\right)}}=\frac{2 \pi}{3}\left[f\left(r^{2}, s^{2}\right)+f\left(0, r^{2}\right)+f\left(s^{2}, 0\right)\right],
$$

which is in fact a minimal cubature formula of degree 2 by (4.6).

On the other hand, a minimal formula of degree 3 has four nodes; it follows from the general theory of minimal cubature formulae that these nodes are common zeros of two orthogonal polynomials of degree 2 ([14]). Since a basis of the orthogonal polynomials with respect to $W_{\Sigma}^{0}$ is known explicitly (cf. Example 3.5), it is not hard to find such a formula. Let $R_{k}^{n}$ denote the orthonormal polynomials of degree $n$ given in Example 3.5 with respect to the weight function $W_{\Sigma}^{0}$. We have

$$
\begin{aligned}
& R_{0}^{2}\left(x_{1}, x_{2}\right)=3\left(3-30 x_{1}+35 x_{1}^{2}\right) / 8 \\
& R_{1}^{2}\left(x_{1}, x_{2}\right)=3 \sqrt{5}\left(-1+7 x_{1}\right)\left(-1+x_{1}+2 x_{2}\right) / 4, \\
& R_{2}^{2}\left(x_{1}, x_{2}\right)=3 \sqrt{35}\left(1-2 x_{1}-8 x_{2}+x_{1}^{2}+8 x_{1} x_{2}+8 x_{2}^{2}\right) / 8,
\end{aligned}
$$

where we take the subscript of $R_{k}^{n}$ as $k=0,1,2$ instead of $k=1,2,3$ as in Example 3.5. The two polynomials of degree 2 that have four common zeros are elements of the subspace spanned by these three polynomials. It turns out, however, that $R_{0}^{2}$ and $R_{2}^{2}$ have four common zeros. We denote these zeros by $\left(s_{i}, t_{i}\right), 1 \leq i \leq 4$; they yield a minimal cubature formula of degree 3 in the form

$$
\int_{\Sigma^{2}} f\left(x_{1}, x_{2}\right) W_{\Sigma}^{0}\left(x_{1}, x_{2}\right) d x_{1} d x_{2}=2 \pi \sum_{i=1}^{4} \lambda_{i} f\left(s_{i}, t_{i}\right)
$$

where the nodes $\left(s_{i}, t_{i}\right)$ and the weights $\lambda_{i}$ are given by

$$
\begin{aligned}
& s_{1}=s_{2}=(15+2 \sqrt{30}) / 35, \quad s_{3}=s_{4}=(15-2 \sqrt{30}) / 35, \\
& t_{1}=(10-\sqrt{30}-\sqrt{65-10 \sqrt{30}}) / 35, \quad t_{2}=(10-\sqrt{30}+\sqrt{65-10 \sqrt{30}}) / 35, \\
& t_{3}=(10+\sqrt{30}-\sqrt{65+10 \sqrt{30}}) / 35, \quad t_{4}=(10+\sqrt{30}+\sqrt{65+10 \sqrt{30}}) / 35 \\
& \lambda_{1}=\lambda_{2}=(18-\sqrt{30}) / 72, \quad \lambda_{3}=\lambda_{4}=(18+\sqrt{30}) / 72,
\end{aligned}
$$


and $t_{1}, t_{2}, t_{3}, t_{4}$ are exactly the zeros of the polynomial $1225 t^{4}-1400 t^{3}+410 t^{2}-40 t+1$. We found the nodes and the weights with the help of the computer program Mathematica. For more on the minimal cubature formula, we refer to [14, 15, 21, 22] and the references there. Since all nodes of (4.11) are located inside the triangle $\Sigma^{2}$, it yields a $\mathbb{Z}_{2} \times \mathbb{Z}_{2} \times \mathbb{Z}_{2}$ invariant cubature formula of degree 7 with 32 nodes,

$$
\int_{S^{2}} f\left(x_{1}, x_{2}, x_{3}\right) d \omega=\frac{\pi}{2} \sum_{i=1}^{4} \lambda_{i} \sum f\left( \pm \sqrt{s_{i}}, \pm \sqrt{t_{i}}, \pm \sqrt{1-s_{i}-t_{i}}\right)
$$

where the inner sum is over all possible choices of signs. Both cubature formulae (4.11) and (4.12) appear to be new. Since $N_{7}^{\min }\left(S^{2}\right)=20$ by (4.8), the formula (4.12) is far from a minimal formula. In [1] or [17, p. 299, formula $\left.U_{3}: 7-2\right]$, a $\mathbb{Z}_{2} \times \mathbb{Z}_{2} \times \mathbb{Z}_{2}$ invariant formula of degree 7 with 26 nodes is given, which yields via Theorem 4.1, however, a cubature formula of degree 3 on $\Sigma^{2}$ with 7 nodes.

In order to construct a $\mathbb{Z}_{2} \times \mathbb{Z}_{2} \times \mathbb{Z}_{2}$ invariant cubature formula on $S^{2}$ with respect to surface measure, we only need to work with a cubature formula with respect to $W_{\Sigma}^{0}$. Several methods used to construct cubature formulae for the Lebesgue measure on $\Sigma^{2}$ may be extended to construct cubature formulae for the weight function $W_{\Sigma}^{0}$, including the method of constructing minimal formulae. At the moment, there is a better understanding of the structure of the cubature formula on the simplex, especially the minimal cubature, and less of the structure of cubature formulae on the sphere. Using Theorem 4.1 in both directions, a number of new cubature formulae on $S^{d}$ as well as on $\Sigma^{d}$ can be constructed. We will present the bulk of the new formulae obtained through Theorem 4.1 in [7], where, among others, formulae on $S^{2}$ that are invariant under the octahedral group (called fully symmetric formulae in $[9,17]$ ) will be constructed by considering symmetric formulae on the triangle $\Sigma^{2}$ (see [13]).

As suggested by Theorem 3.4 and Sobolev's principle, there is also a correspondence between cubature formulae on the simplex $\Sigma^{d}$ and the cubature formulae on the unit ball $B^{d}$, which we now describe. Let us denote by $\mathbb{R}_{+}^{d}$ the positive quadrant of $\mathbb{R}^{d}$; that is, if $\mathbf{x}=\left(x_{1}, \ldots, x_{d}\right) \in \mathbb{R}_{+}^{d}$, then $x_{i} \geq 0$ for $1 \leq i \leq d$. For $\mathbf{x} \in \mathbb{R}_{+}^{d}$, we define $\mathbf{x}^{1 / 2} \in \mathbb{R}_{+}^{d}$ by $\mathbf{x}^{1 / 2}=\left(\sqrt{x_{1}}, \ldots, \sqrt{x_{d}}\right)$.

Theorem 4.5. Let $U$ be a weight function defined on $\mathbb{R}^{d}$ and $U_{\Sigma}$ be defined as in (3.5). If there is a cubature formula of degree $M$ on $\Sigma^{d}$ giving by

$$
\int_{\Sigma^{d}} f(\mathbf{u}) U_{\Sigma}(\mathbf{u}) d \mathbf{u}=\sum_{i=1}^{N} \lambda_{i} f\left(\mathbf{u}_{i}\right), \quad f \in \Pi_{M}^{d}
$$

with all $\mathbf{u}_{i} \in \mathbb{R}_{+}^{d}$, then there is a cubature formula of degree $2 M+1$ on the unit ball $B^{d}$,

$$
\int_{B^{d}} g(\mathbf{x}) U\left(x_{1}^{2}, \ldots, x_{d}^{2}\right) d \mathbf{x}=\sum_{i=1}^{N} \lambda_{i} \sum_{a \in G} g\left(\mathbf{u}_{i}^{1 / 2} a\right) /\left|G\left(\mathbf{u}_{i}\right)\right|, \quad g \in \Pi_{2 M+1}^{d}
$$


Moreover, a cubature formula of degree $2 M+1$ in the form of (4.14) implies a cubature formula of degree $M$ in the form of (4.13).

The proof of this theorem follows exactly as that of Theorem 4.1 if we use the basic formula (2.7) instead of (2.6). We notice that (4.13) is the same as (4.1), except that we no longer require its nodes to be on $\Sigma^{d}$. If a node of $(4.13)$ is not on $\Sigma^{d}$, then the corresponding nodes of (4.14) will be outside of $B^{d}$. Let $N_{k}$ be the number of nodes of (4.13) which lie on $k$-dimensional faces of $\Sigma^{d}$ as in (4.5). Then we also have

Corollary 4.6. Let $N_{M}\left(\Sigma^{d}\right)$ and $N_{2 M+1}\left(B^{d}\right)$ denote the number of nodes of the formula (4.13) and the formula (4.14), respectively. Then the following relation holds

$$
N_{M}\left(\Sigma^{d}\right)=N_{0}+N_{1}+\ldots N_{d} \quad \Longleftrightarrow \quad N_{2 M+1}\left(B^{d}\right)=2^{d} N_{d}+\ldots+2 N_{1}+N_{0} .
$$

A corollary similar to that of Corollary 4.3 also holds. We note that in the correspondence of Theorem 4.5, the Lebesgue measure on $\Sigma^{d}$ corresponds to the measure $\left|x_{1} \cdots x_{d}\right| d \mathbf{x}$ on $B^{d}$, and the Lebesgue measure on $B^{d}$ corresponds to the measure $d \mathbf{u} / \sqrt{u_{1} \cdots u_{d}}$ on $\Sigma^{d}$. In view of Theorem 4.1 , another interesting case is the correspondence between $d \mathbf{x} / \sqrt{1-|\mathbf{x}|^{2}}$ on $B^{d}$ and $d \mathbf{u} /\left(u_{1} \cdots u_{d}\left(1-|\mathbf{u}|_{1}\right)\right)^{1 / 2}$ on $\Sigma^{d}$.

Example 4.7. Let us consider the cubature formula of degree 2 in (4.10) of Example 4.4; by Theorem 4.5 we can transform it to

$$
\int_{B^{2}} f\left(x_{1}, x_{2}\right) \frac{d x_{1} d x_{2}}{\sqrt{1-x_{1}^{2}-x_{2}^{2}}}=\frac{\pi}{6}\left[\sum f( \pm r, \pm s)+\sum f(0, \pm r)+\sum f( \pm s, 0)\right]
$$

where the summations are over all possible choices of signs. This is a cubature formula of degree 5 with 8 nodes for $W_{B}(\mathbf{x})=1 / \sqrt{1-|\mathbf{x}|^{2}}$. The number of nodes of this formula is one more than the lower bound (4.7); we have remarked that (4.7) holds for cubature formulae with respect to $W_{B}$ on $B^{2}$. Using Theorem 4.5, we also conclude that the cubature formula (4.11) yields a $\mathbb{Z}_{2} \times \mathbb{Z}_{2} \times \mathbb{Z}_{2}$ invariant cubature formula of degree 7 with respect to $W_{B}$ on $B^{2}$ with 16 nodes.

\section{REFERENCES}

1. J. Albrecht and L. Collatz,, Zur numerischen auswertung mehrdimensionaler Integrale, Z. Angew. Math. Mech. 38 (1958), 1-15.

2. H. Berens and H. J. Schmid, On the number of nodes of odd degree cubature formulae for integrals with Jacobi weights on a simplex, Numerical Integration (T. O. Espelid and A. Genz, ed.), NATO ASI Series, vol. 367, Kluwer Acad. Publisher, 1992, pp. 37-44.

3. C. Dunkl, Orthogonal polynomials with symmetry of order three, Can. J. Math. 36 (1984), 685-717.

4. C. Dunkl, Orthogonal polynomials on the sphere with octahedral symmetry, Trans. Amer. Math. Soc. 282 (1984), 555-575.

5. C. Dunkl, Differential-difference operators associated to reflection groups, Trans. Amer. Math. Soc. 311 (1989), 167-183.

6. H. Engles, Numerical quadrature and cubature, Academic Press, New York, 1980.

7. S. Heo and Y. Xu, Constructing cubature formulae for the sphere and for the triangle, to appear.

8. E.G. Kalnins, W. Miller, Jr., and M. V. Tratnik, Families of orthogonal and biorthogonal polynomials on the $n$-sphere, SIAM J. Math. Anal. 22 (1991), 272-294. 
9. P. Keast and J. C. Diaz, Fully symmetric integration formulas for the surface of the sphere in $s$ dimensions, SIAM J. Numer. Anal. 20 (1983), 406-419.

10. T. Koornwinder, Two-variable analogues of the classical orthogonal polynomials, Theory and Application of Special Functions (R. A. Askey, ed.), Academic Press, New York, 1975, pp. 435-495.

11. V.I. Lebedev, Quadrature on a sphere, USSR Comp. Math. and Math. Phys. 16 (1976), 10-24.

12. J.N. Lyness and R. Cools, A survey of numerical cubature over triangles, Mathematics of Computation 1943-1993: a half-century of computational mathematics (Vancouver, BC, 1993), Proc. Sympos. Appl. Math., 48, Amer. Math. Soc., Providence, RI, 1994, pp. 127-150.

13. J.N. Lyness and D. Jespersen, Moderate degree symmetric quadrature rules for the triangle, J. Inst. Math. Anal. Appl. 15 (1975), 19-32.

14. H. M. Möller, Kubaturformeln mit minimaler Knotenzahl, Numer. Math. 35 (1976), 185-200.

15. I. P. Mysovskikh, Interpolatory cubature formulas, "Nauka", Moscow, 1981. (Russian)

16. S. L. Sobolev, Cubature formulas on the sphere invariant under finite groups of rotations, Sov. Math. Dokl. 3 (1962), 1307-1310.

17. A. Stroud, Approximate calculation of multiple integrals, Prentice Hall, Englewood Cliffs, NJ, 1971.

18. H. Szegö, Orthogonal polynomials, 4th ed., Amer. Math. Soc. Colloq. Publ. vol.23, Providence, RI, 1975.

19. N. J. Vilenkin, Special functions and the theory of group representations, Amer. Math. Soc. Transl. of Math. Monographs, vol. 22, Providence, RI, 1968.

20. Y. Xu, On zeros of multivariate quasi-orthogonal polynomials and Gaussian cubature formulae, SIAM J. Math. Anal. 25 (1994), 991-1001.

21. Y. Xu, On a class of bivariate orthogonal polynomials and cubature formula, Numer. Math. 69 (1994), 233-241.

22. Y. Xu, Common zeros of polynomials in several variables and higher dimensional quadrature, Pitman Research Notes in Mathematics Series, Longman, Essex, 1994.

23. Y. Xu, On orthogonal polynomials in several variables, Special functions, $q$-series, and related topics, Fields Institute Communications, vol. 14, 1997, pp. 247-270.

24. Y. Xu, Orthogonal polynomials for a family of product weight functions on the spheres, Canadian J. Math. 49 (1997), 175-192.

25. Y. Xu, Orthogonal polynomials and cubature formulae on spheres and on balls, SIAM J. Math. Anal. (to appear).

Yuan Xu, Department of Mathematics, University of Oregon, Eugene, Oregon 974031222 .

E-mail address: yuan@math.uoregon.edu 